\title{
Effectiveness and safety of meropenem/ clavulanate-containing regimens in the treatment of MDR- and XDR-TB
}

\author{
Simon Tiberi ${ }^{1,30}$, Marie-Christine Payen ${ }^{2,30}$, Giovanni Sotgiu ${ }^{3,30}$, \\ Lia D'Ambrosio ${ }^{4,5,30}$, Valentina Alarcon Guizado ${ }^{6}$, Jan Willem Alffenaar ${ }^{7}$, \\ Marcos Abdo Arbex ${ }^{8,9}$, Jose A. Caminero ${ }^{10,11}$, Rosella Centis ${ }^{4}$, \\ Saverio De Lorenzo ${ }^{12}$, Mina Gaga ${ }^{13}$, Gina Gualano ${ }^{14}$, Aurora Jazmín Roby Arias ${ }^{15}$, \\ Anna Scardigli ${ }^{11}$, Alena Skrahina ${ }^{16}$, Ivan Solovic ${ }^{17}$, Giorgia Sulis ${ }^{18}$ \\ Marina Tadolini ${ }^{19}$, Onno W. Akkerman ${ }^{20}$, Edith Alarcon Arrascue ${ }^{11,21}$, \\ Alena Aleska ${ }^{22}$, Vera Avchinko ${ }^{16}$, Eduardo Henrique Bonini, 8,9 , \\ Félix Antonio Chong Marín ${ }^{15}$, Lorena Collahuazo López ${ }^{15}$, Gerard de Vries ${ }^{23}$ \\ Simone Dore ${ }^{3}$, Heinke Kunst ${ }^{24}$, Alberto Matteelli ${ }^{18}$, Charalampos Moschos ${ }^{13}$, \\ Fabrizio Palmieri ${ }^{14}$, Apostolos Papavasileiou ${ }^{13}$, Antonio Spanevello ${ }^{25,26}$, \\ Dante Vargas Vasquez ${ }^{27}$, Pietro Viggiani ${ }^{12}$, Veronica White ${ }^{28}$, Alimuddin Zumla ${ }^{29}$ \\ and Giovanni Battista Migliori ${ }^{4}$
}

ABSTRACT No large study has ever evaluated the efficacy, safety and tolerability of meropenem/ clavulanate to treat multidrug- and extensively drug-resistant tuberculosis (MDR- and XDR-TB). The aim of this observational study was to evaluate the therapeutic contribution, effectiveness, safety and tolerability profile of meropenem/clavulanate added to a background regimen when treating MDR- and XDR-TB cases.

Patients treated with a meropenem/clavulanate-containing regimen $(\mathrm{n}=96)$ showed a greater drug resistance profile than those exposed to a meropenem/clavulanate-sparing regimen $(n=168)$ : in the former group XDR-TB was more frequent $(49 \%$ versus $6.0 \%, \mathrm{p}<0.0001)$ and the median (interquartile range (IQR)) number of antibiotic resistances was higher (8 (6-9) versus 5 (4-6)). Patients were treated with a meropenem/clavulanate-containing regimen for a median (IQR) of 85 (49-156) days.

No statistically significant differences were observed in the overall MDR-TB cohort and in the subgroups with and without the XDR-TB patients; in particular, sputum smear and culture conversion rates were similar in XDR-TB patients exposed to meropenem/clavulanate-containing regimens $(88.0 \%$ versus $100.0 \%, \mathrm{p}=1.00$ and $88.0 \%$ versus $100.0 \%, \mathrm{p}=1.00$, respectively). Only six cases reported adverse events attributable to meropenem/clavulanate (four of them then restarting treatment).

The nondifferent outcomes and bacteriological conversion rate observed in cases who were more severe than controls might imply that meropenem/clavulanate could be active in treating MDR- and XDR-TB cases.

@ERSpublications

Meropenem/clavulanate is effective and safe to treat MDR- and XDR-TB in comparison with controls http://ow.ly/XG75j 
Affiliations: ${ }^{1}$ Division of Infection, Barts Healthcare NHS Trust, London, UK. ${ }^{2}$ Division of Infectious Diseases, CHU Saint-Pierre, Université Libre de Bruxelles (ULB), Brussels, Belgium. ${ }^{3}$ Clinical Epidemiology and Medical Statistics Unit, Dept of Biomedical Sciences, University of Sassari - Research, Medical Education and Professional Development Unit, AOU Sassari, Sassari, Italy. ${ }^{4} \mathrm{WHO}$ Collaborating Centre for TB and Lung Diseases, Fondazione S. Maugeri, Tradate, Italy. ${ }^{5}$ Public Health Consulting Group, Lugano, Switzerland. ${ }^{6}$ National Tuberculosis Control Programme, Ministry of Health, Lima, Peru. ${ }^{7}$ Dept of Clinical Pharmacy and Pharmacology, University Medical Center Groningen, University of Groningen, Groningen, The Netherlands. ${ }^{8}$ University Center of Araraquara, Sao Paulo, Brazil. ${ }^{9}$ Hospital Nestor Goulart Reis, Sao Paulo State Secretary of Health, Sao Paulo, Brazil. ${ }^{10}$ Pneumology Dept, Hospital General de Gran Canaria "Dr. Negrin", Las Palmas de Gran Canaria, Spain. ${ }^{11}$ MDR-TB Unit, Tuberculosis Division, International Union Against Tuberculosis and Lung Disease (The Union), Paris, France. ${ }^{12}$ AOVV E. Morelli Hospital, Reference Hospital for MDR and HIV-TB, Sondalo, Italy. ${ }^{13}$ MDR-TB Unit, Athens Chest Hospital Sotiria, National Referral Centre for Mycobacteria, Athens, Greece. ${ }^{14}$ Respiratory Infectious Diseases Unit, National Institute for Infectious Diseases “L. Spallanzani”, Rome, Italy. ${ }^{15}$ National Tuberculosis Control Programme, Pulmonology Hospital Alfredo J. Valenzuela, Guayaquil, Ecuador. ${ }^{16}$ Republican Research and Practical Centre for Pulmonology and Tuberculosis, Minsk, Belarus. ${ }^{17}$ National Institute for TB, Lung Diseases and Thoracic Surgery, Vysne Hagy, Slovakia. ${ }^{18}$ University Dept of Infectious and Tropical Diseases, WHO Collaborating Centre for TB/HIV Coinfection and for TB Elimination - University of Brescia and Brescia Spedali Civili General Hospital, Brescia, Italy. ${ }^{19}$ Unit of Infectious Diseases, Dept of Medical and Surgical Sciences, Alma Mater Studiorum University of Bologna, Bologna, Italy. ${ }^{20}$ Dept of Pulmonary Diseases and Tuberculosis, TB Center Beatrixoord, University Medical Center Groningen, University of Groningen, Groningen, The Netherlands. ${ }^{21}$ International Union Against Tuberculosis and Lung Disease (The Union), Lima, Peru. ${ }^{22}$ Educational Institution "Grodno State Medical University", Grodno, Belarus. ${ }^{23}$ KNCV Tuberculosis Foundation, The Hague, The Netherlands. ${ }^{24} \mathrm{Dept}$ of Respiratory Medicine, Queen Mary University, London, UK. ${ }^{25}$ Pneumology Unit, Fondazione Maugeri, IRCCS, Tradate, Italy. ${ }^{26}$ Dept of Clinical and Experimental Medicine, University of Insubria, Varese, Italy. ${ }^{27}$ National Hospital Hipólito Unanue, Public Health Institute, Ministry of Health, Lima, Peru. ${ }^{28}$ Dept of Respiratory Medicine, Barts Healthcare NHS Trust, London, UK. ${ }^{29}$ Division of Infection and Immunity, University College London and NIHR Biomedical Research Centre, UCL Hospitals NHS Foundation Trust, London, UK. ${ }^{30}$ These authors contributed equally to this work.

Correspondence: Giovanni Battista Migliori, World Health Organization Collaborating Centre for Tuberculosis and Lung Diseases, Fondazione S. Maugeri, Care and Research Institute, Via Roncaccio 16, 21049 Tradate, Italy.

E-mail: giovannibattista.miglioriafsm.it

\section{Introduction}

The World Health Organization (WHO) estimated that 480000 new multidrug-resistant tuberculosis (MDR-TB) cases occurred globally in 2014, with 190000 deaths [1]. Only one MDR-TB case out of three is currently diagnosed and one out of four treated [1]. Of the 123000 cases of MDR-TB notified to the WHO in 2014, about half were detected in India, the Russian Federation and South Africa. Overall, 3.3\% of new cases and $20 \%$ of previously treated cases of TB are MDR-TB. In Belarus, over $50 \%$ of TB cases are MDR-TB; $34 \%$ of new cases and $64 \%$ of those previously treated $[1,2]$. The proportion of MDR-TB cases harbouring extensively drug-resistant (XDR)-TB strains of Mycobacterium tuberculosis in 2014 was highest in Belarus (29\%), Georgia (15\%) and Latvia (19\%), and accounted for up to 9.7\% globally [1, 2].

Treatment of MDR-TB cases is long, expensive and associated with frequent adverse events [3-8]. Furthermore, a large proportion of patients with MDR-TB have additional drug resistances and this makes the choice of an adequate treatment very difficult.

The WHO guidelines in force recommend the stepwise use of second-line drugs classified into five groups [7], although modifications to drug grouping have been proposed recently [9] and discussion is ongoing at the WHO level.

The difficulty in identifying at least four active drugs suitable for inclusion in a multidrug regimen effective against these resistant forms of TB makes the need for new antibiotics urgent $[3,4,7,10,11]$.

Presently, there is a lot of interest in new drugs such as delamanid [12-14] and bedaquiline [15-20], and in repurposed drugs, which are included in WHO Group 5, i.e. drugs with unknown/limited evidence on efficacy and/or tolerability [7, 21]. This group includes linezolid [10, 22-28] and carbapenems [29-34]. Carbapenems represent an interesting class of drugs (including meropenem, imipenem and ertapenem), which are widely used to treat MDR- and XDR-TB cases, although very limited evidence exists regarding their efficacy, safety and tolerability [29-34].

The association of a $\beta$-lactam antibiotic with a $\beta$-lactamase inhibitor was explored recently with apparently suboptimal results, as Mycobacterium tuberculosis is protected from $\beta$-lactam antibiotics through its potent $\beta$-lactamase, encoded by the blaC gene $[35,36]$. Clavulanate (a $\beta$-lactamase inhibitor) has a demonstrated capacity to inhibit the activity of blaC-coded products in vitro [36]. Meropenem, a carbapenem offering a limited substrate for hydrolysis, has demonstrated high bactericidal in vitro activity when combined with clavulanate against susceptible, MDR- and XDR-TB strains of M. tuberculosis, and the ability to sterilise 
cultures in vivo within 2 weeks [37, 38]. It has also shown promising activity in a murine model [39] and its clinical effectiveness against $M$. tuberculosis-related disease was proved in two case reports of 10 cases or less $[30,40]$. To date, a single study is available in the literature suggesting the drug is effective and well tolerated. Unfortunately, this study had a limited sample size of 37 patients recruited at a single centre and was focused on the evaluation of bacteriological conversion, without providing final treatment outcomes [29].

Taking advantage of existing collaborations and established research protocols, TB reference centres belonging to the International Carbapenems Study Group (ICSG) [31] conducted a large observational study to evaluate the therapeutic contribution, effectiveness, safety and tolerability profile of meropenem/ clavulanate added to a background regimen (as per the WHO guidelines) when treating MDR- and XDR-TB cases.

\section{Material and methods}

An observational, retrospective, cohort study was performed in five centres specialised in the management of MDR- and XDR-TB cases in three countries which decided to adhere to the ICSG project.

Individuals aged $<15$ years were excluded. Only adults with a culture-confirmed diagnosis of MDR-TB (i.e. TB caused by $M$. tuberculosis isolates resistant to at least isoniazid and rifampicin) were enrolled and evaluated according to meropenem/clavulanate exposure (the time period of clinical evaluation ranged from 2003 to 2015).

Individuals treated with MDR-TB meropenem/clavulanate-containing and -sparing regimens were consecutively recruited following a retrospective design in all centres. Specifically, patients from each participating centre were separated in groups exposed and not exposed to meropenem/clavulanate.

Anti-TB drugs were prescribed following the WHO recommendations based on drug-susceptibility test (DST) results provided by quality-assured laboratories serving all the participating centres without any blinding or randomisation procedures [11]. Meropenem/clavulanate was administered off-label at a dosage of $1 \mathrm{~g}$ three times daily intravenously ( $2 \mathrm{~g}$ three times daily in Belgium) and clavulanate in the form of oral amoxicillin/ clavulanic acid. The ratio between those exposed and not exposed to meropenem/clavulanate-containing regimens was 1:2.

Demographic, epidemiological, clinical and microbiological variables were retrospectively collected from medical records using standardised ad hoc E-forms. In particular, the following information was retrieved: date of admission, length of hospital stay, date and place of birth, sex, residence, immigration from a high TB burden country, HIV status, exposure to antiretroviral drugs, previous TB diagnoses, previous anti-TB treatments (i.e. exposure to anti-TB drugs for $>1$ month) and previous treatment outcomes, DST results, including susceptibility or resistance to the drugs defining XDR-TB (i.e. TB caused by $M$. tuberculosis strains resistant to isoniazid, rifampicin, any fluoroquinolones and at least a first-line injectable anti-TB drug: amikacin, capreomycin or kanamycin), radiological findings, anti-TB regimen administered (including dosage and length of exposure), surgery, duration of exposure to meropenem/clavulanate and other relevant drugs (e.g. linezolid) and adverse events potentially ascribed to the meropenem/clavulanate and other relevant drugs (e.g. linezolid), management of adverse events, proportion of sputum smear and culture positivity at the hospital admission and at 30,60 and 90 days after the prescription of second-line anti-TB drugs, time to sputum smear/culture conversion, and treatment outcomes.

A descriptive analysis of the qualitative and quantitative variables was performed using absolute and relative (percentages) frequencies and mean \pm SD or median (interquartile range (IQR)) according to their parametric distribution, respectively. Inference analysis was carried out with Chi-squared or Fisher's exact tests and the Wilcoxon-Mann-Whitney test for categorical and numerical variables, respectively. Survival analyses were performed to assess the microbiological (i.e. sputum smear and culture) conversion in patients treated with meropenem/clavulanate-containing and -sparing regimens.

Two-tailed $p$-values were deemed statistically significant when $p<0.05$. All statistical analyses were carried out with Stata 13.0 statistical software (StataCorp, College Station, TX, USA).

Ethical approval for the collection and analysis of anonymous and retrospective data and for the compassionate use of the drugs was obtained by the institutional review boards of the participating institutions as per legislation in force in the different ICSG countries and at the coordinating centre.

\section{Results}

A retrospective cohort of 264 MDR-TB patients was recruited (table 1). 21.6\% were affected by XDR-TB.

96 were treated with meropenem/clavulanate-containing and 168 with meropenem/clavulanate-sparing regimens. The majority (52.1\%) of the patients were males, with a median (IQR) age of 33 (26-43) years, 
TABLE 1 Demographic, epidemiological and clinical characteristics of multidrug- and extensively drug-resistant tuberculosis (MDR- and XDR-TB) patients treated with optimised background regimen with and without additional meropenem/clavulanate (MC)

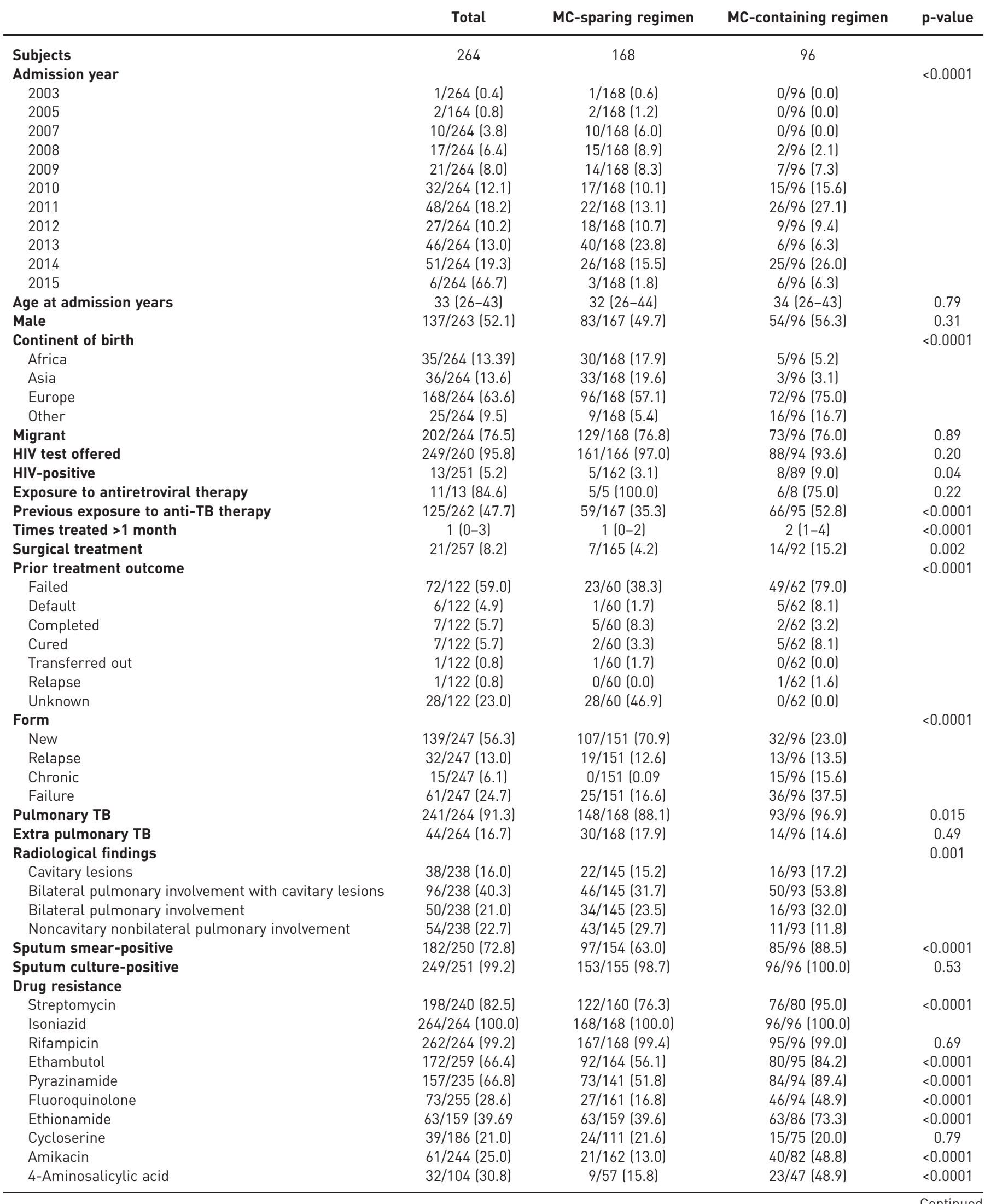


TABLE 1 Continued

\begin{tabular}{|c|c|c|c|c|}
\hline & Total & MC-sparing regimen & MC-containing regimen & p-value \\
\hline Capreomycin & $55 / 207(26.6)$ & $17 / 126(13.5)$ & $38 / 81(46.9)$ & $<0.0001$ \\
\hline Kanamicin & $53 / 163(32.5)$ & $16 / 88$ (18.2) & $37 / 75(49.3)$ & $<0.0001$ \\
\hline Rifabutin & $108 / 137(78.8)$ & $83 / 111(74.8)$ & $25 / 26(96.2)$ & 0.02 \\
\hline Other & 9/27 (33.3) & $3 / 16(18.8)$ & $6 / 11(54.6)$ & 0.10 \\
\hline Antibiotic resistances & $6(4-8)$ & $5(4-6)$ & $8(6-9)$ & $<0.0001$ \\
\hline XDR & $57 / 264(21.6)$ & $10 / 168(6.0)$ & $47 / 96(49.0)$ & $<0.0001$ \\
\hline Moxifloxacin & $210 / 254(82.7)$ & $133 / 159(83.7)$ & $77 / 95(81.1)$ & 0.60 \\
\hline Levofloxacin & $35 / 261(13.4)$ & $26 / 165(15.8)$ & $9 / 96(9.4)$ & 0.15 \\
\hline Linezolid & $74 / 188(39.4)$ & $24 / 98(24.5)$ & $50 / 90(55.6)$ & $<0.0001$ \\
\hline Delamanid & 1/239 (0.4) & $0 / 144(0.0)$ & 1/95 (1.1) & 0.40 \\
\hline Bedaquiline & $11 / 239(4.6)$ & $2 / 144(1.4)$ & 9/95 (9.5) & 0.008 \\
\hline
\end{tabular}

Data are presented as $\mathrm{N}$ or $\mathrm{n} / \mathrm{N}(\%)$ or median (interquartile range), unless otherwise stated. Denominators (N) were different among the selected variables because of missing data.

and most of them were migrants $(76.5 \%)$, whose country of birth was located in Europe in the majority of the cases (61.4\%). 13 individuals (5.2\%) were HIV-positive. Most cases (91.3\%) were affected by pulmonary $\mathrm{TB}$, with percentages of sputum smear and culture confirmation of 72.8 and $99.2 \%$, respectively. Almost half of the cases $(47.7 \%)$ were previously treated (in the majority of the cases for drug-susceptible TB, according to the medical history); the commonest previous treatment outcome was failure $(59.0 \%)$ and $8.2 \%$ underwent surgery.

Patients treated with a meropenem/clavulanate-containing regimen showed a greater drug resistance profile than those exposed to a meropenem/clavulanate-sparing regimen: in the former group, XDR-TB was more frequent $(49 \%$ versus $6.0 \%, \mathrm{p}<0.0001)$ and the median (IQR) number of antibiotic resistances was higher (8 (6-9) versus 5 (4-6)).

Patients were treated with a meropenem/clavulanate-containing regimen for a median of 85 (49-156) days (table 2). No statistically significant differences in terms of effectiveness indicators were observed in the

TABLE 2 Safety and tolerability in multidrug- and extensively drug-resistant tuberculosis patients exposed and not exposed to meropenem/clavulanate (MC)-containing regimens

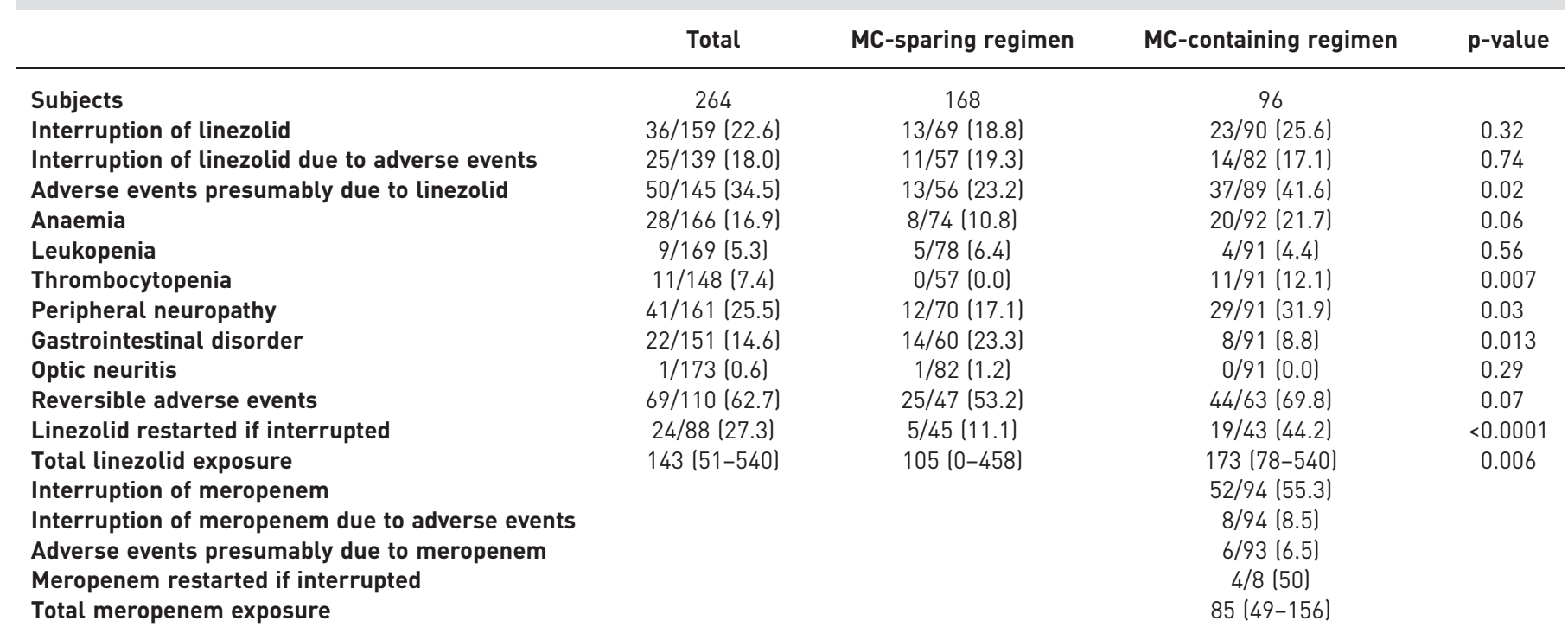

Data are presented as $\mathrm{N}$ or $\mathrm{n} / \mathrm{N}(\%)$ or median (interquartile range), unless otherwise stated. Denominators $(\mathrm{N})$ were different among the selected variables because of missing data. 
overall MDR-TB cohort and in the subgroups with and without the XDR-TB patients; in particular, sputum smear and culture conversion rates were similar in XDR-TB patients exposed to meropenem/ clavulanate-containing regimens $(88.0 \%$ versus $100.0 \%, \mathrm{p}=1.00$ and $88.0 \%$ versus $100.0 \%, \mathrm{p}=1.00$, respectively) (table 3 and figure 1$)$.

A subanalysis performed after stratifying all MDR-TB cases for $n<6$ or $n \geqslant 6$ drug resistances (median number of drug resistances in the entire cohort) did not identify any statistically significant differences in sputum smear and culture conversion rates and treatment success rates between the two arms, with the only exception of the higher treatment success in those with $\mathrm{n}<6$ drug resistances and treated with a meropenem/clavulanate-sparing regimen.

Only six cases reported adverse events that could be attributable to meropenem/clavulanate and four of them restarted treatment after its interruption (table 2).

\section{Discussion}

The aim of our study was to evaluate the therapeutic contribution of meropenem/clavulanate when added to a background regimen (as per the WHO guidelines) in terms of effectiveness, safety and tolerability in treating MDR- and XDR-TB cases.

TABLE 3 Treatment outcomes of multidrug- and extensively drug-resistant tuberculosis (MDR- and XDR-TB) patients exposed and not exposed to meropenem/clavulanate (MC)-containing regimens

Total MC-sparing regimen MC-containing regimen $\mathrm{p}$-value

\section{Subjects}

Sputum smear conversion

At 30 days of treatment

At 60 days of treatment

At 90 days of treatment

In patients with $<6$ resistances

In patients with $\geqslant 6$ resistances

In MDR-TB patients

In XDR-TB patients

In MDR-TB patients without resistance to fluoroquinolones

In MDR-TB patients with resistance to fluoroquinolones

\section{Sputum culture conversion}

At 30 days of treatment

At 60 days of treatment

At 90 days of treatment

In patients with $<6$ resistances

In patients with $\geqslant 6$ resistances

In MDR-TB patients

In XDR-TB patients

In MDR-TB patients without resistance to fluoroquinolones

In MDR-TB patients with resistance to fluoroquinolones

Time from start of anti-TB therapy to sputum smear

conversion days

Time from start of anti-TB therapy to culture conversion days

Treatment outcome

Cured

Treatment completed

Still on treatment

Died

Default

Transferred out

\section{Treatment success}

In patients with $<6$ resistances

In patients with $\geqslant 6$ resistances

In MDR-TB patients

In XDR-TB patients

264

$155 / 160$ (96.9)

$128 / 227(56.4)$

$148 / 208$ (71.2)

$169 / 201(84.1)$

$65 / 66$ (98.5)

90/94 (95.7)

$126 / 128(98.4)$

$29 / 32(90.6)$

$142 / 145(97.9)$

$29 / 30(96.7)$

156/159 (98.1)

$100 / 210(47.6)$

$118 / 186(63.4)$

$132 / 180$ (73.3)

$66 / 66(100.0)$

$90 / 93$ (96.8)

$127 / 127$ (100.0)

$29 / 32(90.6)$

$140 / 141$ (99.3)

$29 / 30(96.7)$

$30(7-61)$

$42(29-90)$

$89 / 264$ (33.7)

$81 / 264(30.7)$

$54 / 264(20.5)$

$14 / 264(5.3)$

$21 / 264(8.0)$

$5 / 264$ (1.9)

$170 / 264(64.4)$

$76 / 91$ (83.5)

92/111 (82.9)

$141 / 207(68.1)$

29/57 (50.9)
$100 / 102(98.0)$

$91 / 133$ (68.4)

89/117 (76.1)

93/111 (83.8)

$56 / 57$ (98.3)

44/45 (97.8)

93/95 (97.9)

$7 / 7$ (100.0)

$107 / 110$ (97.3)

$17 / 18$ (94.4)

$101 / 101$ (100.0)

$70 / 116$ (60.3)

$59 / 93(63.4)$

60/89 (67.4)

$57 / 57$ (100.0)

44/44 (100.0)

94/94 (100.0)

$7 / 7$ (100.0)

105/106 (99.1)

$17 / 18$ (94.4)

$30(0-56)$

$42(30-90)$

67/168 (39.9)

$48 / 168(28.6)$

$30 / 168$ (17.9)

$3 / 168$ (1.8)

$16 / 168$ (9.5)

$4 / 168(2.4)$

$115 / 168$ (68.5)

$67 / 77$ (87.0)

46/54 (85.2)

$108 / 158(68.4)$

$7 / 10(70.0)$
96

$55 / 58$ (94.8)

$37 / 94(39.4)$

$59 / 91$ (64.8)

76/90 (84.4)

9/9 (100.0)

46/49 (93.9)

$33 / 33(100.0)$

$22 / 25$ (88.0)

$35 / 35$ (100.0)

$12 / 12(100.0)$

$55 / 58$ (94.8)

30/94 (31.9)

$59 / 93(63.4)$

72/91 (79.1)

$9 / 9$ (100.0)

$46 / 49(93.9)$

$33 / 33(100.0)$

$22 / 25(88.0)$

$35 / 35$ (100.0)

$12 / 12(100.0)$

45 (28-68)

0.35

$<0.0001$

0.08

0.90

1.00

0.62

1.00

1.00

1.00

1.00

0.05

$<0.0001$

1.00

0.08

1.00

0.24

1.00

1.00

1.00

1.00

0.0002

44 (28-75)

0.81

0.001

$22 / 96$ (22.9)

$33 / 96(34.4)$

24/96 (25.0)

$11 / 96$ (11.5)

$5 / 96$ (5.2)

$1 / 96$ (1.0)

$55 / 96$ (57.3)

$9 / 14$ (64.3)

46/57 (80.7)

0.07

$33 / 49(67.4)$

0.035

0.53

22/47 (46.8)
0.90

0.30

Data are presented as $\mathrm{N}$ or $\mathrm{n} / \mathrm{N}(\%)$ or median (interquartile range), unless otherwise stated. Denominators (N) were different among the selected variables because of missing data. 

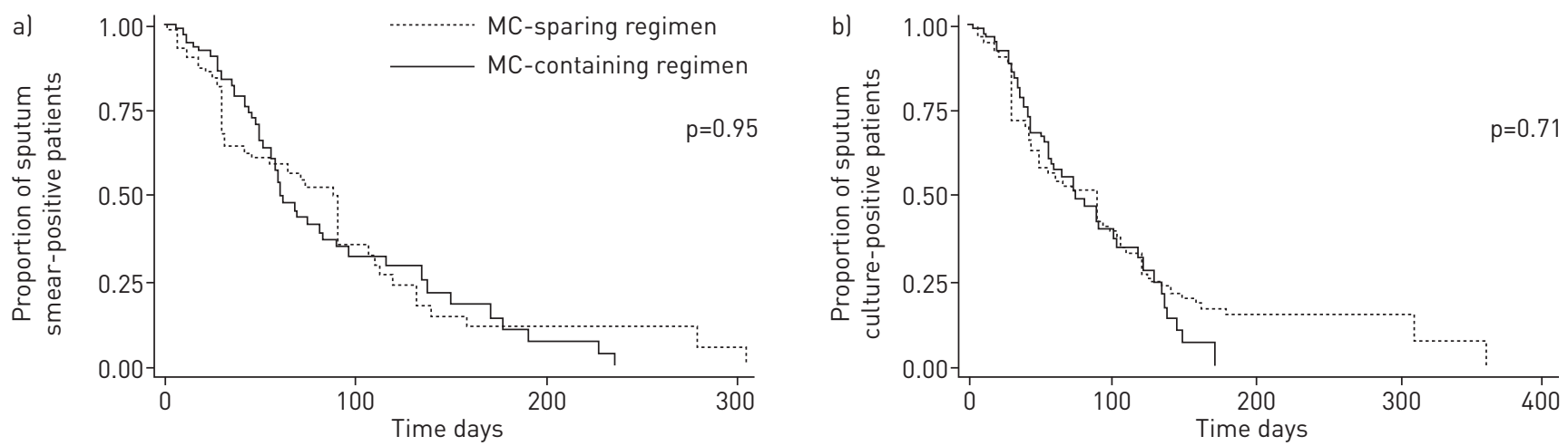

FIGURE 1 a) Sputum smear and b) culture conversion in multidrug- and extensively drug-resistant tuberculosis patients exposed and not exposed to meropenem/clavulanate (MC)-containing regimens.

The results of our study demonstrate that meropenem/clavulanate-containing and -sparing regimens achieve similar sputum smear and culture conversion rates in XDR-TB cases. No statistically significant differences in terms of treatment outcomes were observed between the patients with and without a meropenem/clavulanate-containing regimen, in spite of the fact that the former had a more severe form of disease and a larger number of drug resistances. Furthermore, the safety profile of meropenem/clavulanate (previously demonstrated in the treatment of other bacterial diseases) was confirmed and only two patients did not continue the treatment with meropenem/clavulanate.

This is, to the best of our knowledge, the first large study (sample size >90) on meropenem/clavulanate administered to TB patients in the scientific literature. Among the strengths of the study we mention the large size of the entire cohort, and the detailed information collected from all five centres and three countries in Europe and Latin America.

However, the study shows the following limitations, which are in-built in its observational, retrospective design. 1) Having not been planned prospectively, the computation of a primary hypothesis-based sample size was not allowed. 2) Given each regimen (as per the WHO guidelines) consisted of a cocktail of drugs, it was difficult to attribute a specific effect to a specific drug (meropenem/clavulanate in this case). 3) No randomisation and blinding procedures were performed. 4) As expected, the patients enrolled in the meropenem/clavulanate-containing arm were significantly more complex in terms of drug resistance patterns, while most of the demographic, epidemiological and clinical variables did not differ significantly. In this respect, the nondifferent results in terms of outcomes and bacteriological conversions observed in MDR- and XDR-TB cases might imply that meropenem/clavulanate could be active. This finding needs to be confirmed by controlled clinical trials which will remove the "background noise" produced by the observational design. 5) As no standard DST for meropenem/clavulanate was available, some isolates may have been resistant to the drug. The evidence provided by this study allows a greater understanding of the role of carbapenems and confirms the results of previous studies [29,30]. Meropenem/clavulanate is safe and its activity appears promising, although probably not as active as other repurposed drugs (e.g. linezolid [22-28]).

Furthermore, it should be highlighted that meropenem/clavulanate is expensive as compared with other antibiotics and is not available in all countries, particularly in geographical areas with a high MDR- and XDR-TB burden and limited economic resources.

We note that treatment success in the cohort of the XDR-TB patients treated with meropenem/clavulanate was higher than that achieved in the largest observational cohort available in the literature $(46.8 \%$ versus $40.0 \%$ ) [3, 4]. It will be interesting to compare the results of this study with an ongoing study (by the same international group) on imipenem, which is cheaper and more widely available.

As the future role of carbapenems in the treatment of MDR- and XDR-TB needs further discussion [9, 41], the evidence provided by this observational study offers new information to clinicians treating severely resistant TB cases (e.g. those with resistances to six drugs or more or with intolerance to drugs) and lacking the minimum number of active drugs necessary to design an effective regimen $[3,4]$. For this reason, 12 of the patients treated with meropenem/clavulanate were prescribed bedaquiline $(n=11)$ or delamanid $(n=1)$. Even less evidence is presently available for other drugs which are already in use (e.g. sulphonamides [42, 43] and mefloquine [44]).

Although the combination of meropenem/clavulanate with delamanid and bedaquiline is potentially useful to avoid rapid resistance development to the new compounds, some concerns have been raised about the 
QTc prolongation and related cardiotoxicity. Fortunately, no cases were detected in our study. This toxicity might be further enhanced by other second-line drugs, such as fluoroquinolones or clofazimine [45].

Although observational studies show some methodological shortcomings, they could offer rapid orientation on the drug effectiveness and safety in order to better inform future clinical trials. In the case of linezolid, the observational evidence was confirmed by that generated by a clinical trial [26].

Results of a phase IIa study evaluating the early bactericidal activity of meropenem/clavulanate (NCT02349841) are expected soon and may confirm our results. In addition, the oral carbapenems currently under evaluation (e.g. faropenem (NCT02349841 and NCT02381470)) might shed new light on the use of carbapenems for the treatment of MDR- and XDR-TB.

Moreover, our results suggest that meropenem/clavulanate can improve the outcome in MDR- and XDR-TB, although further studies correlating resistance testing for carbapenems with outcomes are necessary.

Phenotypic/genotyping resistance testing for meropenem/clavulanate susceptibility may assist clinicians in identifying patients who may benefit from treatment of this drug.

MDR-TB is a global public health priority and challenge; the rapid implementation of new clinical trials with repurposed drugs is an important component towards TB elimination that needs to be addressed [46].

\section{References}

World Health Organization. Global Tuberculosis Report 2015. Geneva, World Health Organization, 2015.

2 Skrahina A, Hurevich $\mathrm{H}$, Zalutskaya A, et al. Alarming levels of drug-resistant tuberculosis in Belarus: results of a survey in Minsk. Eur Respir J 2012; 39: 1425-1431.

3 Migliori GB, Sotgiu G, Gandhi NR, et al. Drug resistance beyond extensively drug resistant tuberculosis: individual patient data meta-analysis. Eur Respir J 2013; 42: 169-179.

4 Falzon D, Gandhi N, Migliori GB, et al. Resistance to fluoroquinolones and second-line injectable drugs: impact on multidrug-resistant TB outcomes. Eur Respir J 2013; 42: 156-168.

5 Diel R, Rutz S, Castell S, et al. Tuberculosis: cost of illness in Germany. Eur Respir J 2012; 40: 143-115.

6 Diel R, Vandeputte J, de Vries G, et al. Costs of tuberculosis disease in the European Union: a systematic analysis and cost calculation. Eur Respir J 2014; 43: 554-565.

7 Falzon D, Jaramillo E, Schünemann HJ, et al. WHO guidelines for the programmatic management of drug-resistant tuberculosis: 2011 update. Eur Respir J 2011; 38: 516-528.

8 Migliori GB, De Iaco G, Besozzi G, et al. First tuberculosis cases in Italy resistant to all tested drugs. Euro Surveill 2007; 12: E070517.1.

9 Caminero JA, Scardigli A. Classification of antituberculosis drugs: a new proposal based on the most recent evidence. Eur Respir J 2015; 46: 887-893.

10 Sotgiu G, Pontali E, Migliori GB. Linezolid to treat MDR-/XDR-tuberculosis: available evidence and future scenarios. Eur Respir J 2015; 45: 25-29.

11 Pontali E, Sotgiu G, D'Ambrosio L, et al. Bedaquiline and MDR-TB: a systematic and critical analysis of the evidence. Eur Respir J 2016; 47: 394-402.

12 Esposito S, D’Ambrosio L, Tadolini M, et al. ERS/WHO Tuberculosis Consilium assistance with extensively drug-resistant tuberculosis management in a child: case study of compassionate delamanid use. Eur Respir J 2014; 44: 811-815.

13 Skripconoka V, Danilovits M, Pehme L, et al. Delamanid improves outcomes and reduces mortality in multidrug-resistant tuberculosis Eur Respir J 2013; 41: 1393-1400.

14 Gler MT, Skripconoka V, Sanchez-Garavito E, et al. Delamanid for multidrug-resistant pulmonary tuberculosis. N Engl J Med 2012; 366: 2151-2160.

15 Pym AS, Diacon AH, Tang S-J, et al. Bedaquiline in the treatment of multidrug- and extensively drug-resistant tuberculosis. Eur Respir J 2016; 47: 564-574.

16 Diacon AH, Pym A, Grobusch M, et al. The diarylquinoline TMC207 for multidrug-resistant tuberculosis. $N$ Engl J Med 2009; 360: 2397-2405.

17 Diacon AH, Pym A, Grobusch MP, et al. Multidrug-resistant tuberculosis and culture conversion with bedaquiline. N Engl J Med 2014; 371: 723-732.

18 Guglielmetti L, Le Dû D, Jachym M, et al. Compassionate use of bedaquiline for the treatment of multidrug-resistant and extensively drug-resistant tuberculosis: interim analysis of a French cohort. Clin Infect Dis 2015; 60: 188-194.

19 Ndjeka N, Conradie F, Schnippel K, et al. Treatment of drug-resistant tuberculosis with bedaquiline in a high HIV prevalence setting: an interim cohort analysis. Int J Tuberc Lung Dis 2015; 19: 979-985.

20 Tiberi S, De Lorenzo S, Centis R, et al. Bedaquiline in MDR/XDR-TB cases: first experience on compassionate use. Eur Respir J 2014; 43: 289-292.

21 Tiberi S, D'Ambrosio L, De Lorenzo S, et al. Tuberculosis elimination, patients' lives and rational use of new drugs: revisited. Eur Respir J 2016; 47: 664-667.

22 Migliori GB, Eker B, Richardson MD, et al. A retrospective TBNET assessment of linezolid safety, tolerability and efficacy in MDR-TB. Eur Respir J 2009; 34: 387-393.

23 Villar M, Sotgiu G, D’Ambrosio L, et al. Linezolid safety, tolerability and efficacy to treat multidrug- and extensively drug-resistant tuberculosis. Eur Respir J 2011; 38: 730-733.

24 De Lorenzo S, Centis R, D’Ambrosio L, et al. On linezolid efficacy and tolerability. Eur Respir J 2012; 39: 770-772.

25 Sotgiu G, Centis R, D'Ambrosio L, et al. Efficacy, safety and tolerability of linezolid containing regimens in treating MDR-TB and XDR-TB: systematic review and meta-analysis. Eur Respir J 2012; 40: 1430-1442.

26 Sotgiu G, Centis R, D'Ambrosio L, et al. Linezolid to treat extensively drug-resistant TB: retrospective data are confirmed by experimental evidence. Eur Respir J 2013; 42: 288-290. 
27 Lee M, Lee J, Carroll MW, et al. Linezolid for treatment of chronic extensively drug-resistant tuberculosis. $N$ Engl J Med 2012; 367: 1508-1518.

28 Sotgiu G, Centis R, D'Ambrosio L, et al. Low minimal inhibitory concentrations of linezolid against multidrug-resistant tuberculosis strains. Eur Respir J 2015; 45: 287-289.

29 De Lorenzo S, Alffenaar JW, Sotgiu G, et al. Efficacy and safety of meropenem-clavulanate added to linezolid-containing regimens in the treatment of MDR-/XDR-TB. Eur Respir J 2013; 41: 1386-1392.

30 Payen MC, De Wit S, Martin C, et al. Clinical use of meropenem-clavulanate combination for extensively drug-resistant tuberculosis. Int J Tuberc Lung Dis 2012; 16: 558-560.

31 Tiberi S, D'Ambrosio L, De Lorenzo S, et al. Ertapenem in the treatment of MDR-TB: first clinical experience. Eur Respir J 2016; 47: 333-336.

32 van Rijn SP, van Altena R, Akkerman OW, et al. Pharmacokinetics of ertapenem in patients with multidrug-resistant tuberculosis. Eur Respir J 2016; 47: 1229-1234.

33 Chambers HF, Turner J, Schecter GF, et al. Imipenem for treatment of tuberculosis in mice and humans. Antimicrob Agents Chemother 2005; 49: 2816-2282.

34 Arbex MA, Ribeiro de Siqueira H, D'Ambrosio L, et al. The challenge of managing extensively drug-resistant tuberculosis at a referral hospital in the state of São Paulo, Brazil: a report of three cases. J Bras Pneumol 2015; 41: 554-559.

35 Yew WW, Wong CF, Lee J, et al. Do beta-lactam-beta-lactmase inhibitor combinations have a place in the treatment of multidrug-resistant pulmonary tuberculosis? Tubercle Lung Dis 1995; 78: 90-92.

36 Hugonnet JE, Blanchard JS. Irreversible inhibition of Mycobacterium tuberculosis beta-lactamase by clavulanate. Biochemistry 2007; 46: 11198-12004.

37 Hugonnet JE, Tremblay LW, Boshoff HI, et al. Meropenem-clavulanate is effective against extensively drug-resistant Mycobacterium tuberculosis. Science 2009; 323: 1215-1218.

38 Payen M, Martin C, Antoine-Moussiaux T, et al. Four cases of XDR-TB treated with meropenem-clavulanate. 50th Interscience Conference on Antimicrobial Agents and Chemotherapy (ICAAC), 2010. Abstract L1-517. www. abstractsonline.com/Plan/ViewAbstract.aspx?sKey=7189a738-03a3-41e0-8ee5-cf36269b5766\&cKey=aceae86c-c8ae-4d8e968e-d2dd95abed53\&mKey=\%7b93AEED6A-54D4-4EF6-99BD-A9B3CE9FACD9\%7d Date last accessed: February 23, 2016.

39 Veziris N, Truffot C, Mainardi J-L, et al. Activity of carbapenems combined with clavulanate against murine tuberculosis. Antimicrob Agents Chemother 2011; 55: 2597-2600.

40 Palmero D, Montaner PG, Cufré M, et al. Primera serie de pacientes con TB-XDR y pre-XDR tratados con esquemas que incluyeron meropenem-clavulanato en Argentina. [First series of patients with XDR and Pre-XDR TB treated with regimens that included meropenen-clavulanate in Argentina.] Arch Bronconeumol 2015; 51: e49-e52.

41 Winters N, Butler-Laporte G, Menzies D. Efficacy and safety of World Health Organization group 5 drugs for multidrug-resistant tuberculosis treatment. Eur Respir J 2015; 46: 1461-1470.

42 Alsaad N, Wilffert B, van Altena R, et al. Potential antimicrobial agents for the treatment of multidrug-resistant tuberculosis. Eur Respir J 2014; 43: 884-897.

43 Alsaad N, van Altena R, Pranger AD, et al. Evaluation of co-trimoxazole in the treatment of multidrug-resistant tuberculosis. Eur Respir J 2013; 42: 504-512.

44 Krieger D, Vesenbeckh S, Schönfeld $\mathrm{N}$, et al. Mefloquine as a potential drug against multidrug-resistant tuberculosis. Eur Respir J 2015; 46: 1503-1505.

45 Matteelli A, D'Ambrosio L, Centis R, et al. Compassionate and optimum use of new tuberculosis drugs. Lancet Infect Dis 2015; 15: 1131-1132.

46 Lonnroth K, Migliori GB, Abubakar I, et al. Towards tuberculosis elimination: an action framework for low-incidence countries. Eur Respir J 2015; 45: 928-952. 